\title{
Yield performance of the European Union Maize Landrace Core Collection under multiple corn borer infestations
}

\author{
R.A. Malvar ${ }^{a,}$, A. Butrón ${ }^{a}$, A. Alvarez ${ }^{\text {b, }}$, G. Padilla ${ }^{a}$, M.E. Cartea ${ }^{a}$, P. Revilla ${ }^{a}$ and A. \\ Ordás $^{a}$
}

aMisión Biológica de Galicia, Spanish Council for Scientific Research, Apartado 28, 36080 Pontevedra, Spain

${ }^{\text {b} E s t a c i o ́ n ~ E x p e r i m e n t a l ~ d e ~ A u l a ~ D e i, ~ S p a n i s h ~ C o u n c i l ~ f o r ~ S c i e n t i f i c ~ R e s e a r c h, ~ A p a r t a d o ~}$ 202, 50080 Zaragoza, Spain

\begin{abstract}
In Europe, corn borer attack is the main biotic stressor for the maize (Zea mays L.) crop. European corn borer (Ostrinia nubilalis Hbn.) is the most important maize pest in central and north Europe, while pink stem borer (Sesamia nonagrioides Lef.) is predominant in warmer areas of southern Europe. The objective of this study was the evaluation of the European Maize Union Landrace Core Collection (EUMLCC) for yield under infestation with European corn borer (O. nubilalis) and pink stem borer (S. nonagrioides). Eighty-five landraces from Germany, Spain, France, Greece, Italy, and Portugal were evaluated, under corn borer infestation, for yield, grain moisture, and days to flowering at two locations in Spain. Landraces were evaluated separately in four trials that corresponded to four maturity groups. In each maturity group, there were significant differences among landraces for yield of infested plants. Extra-early landraces, ESP0090214, FRA0410010, and ESP0070339; early landraces, FRA0410022, and ESP11985022; midseason landraces, PRT00100392 and ESP11981047; and late landraces, PRT00100569 and
\end{abstract}


PRT00100530, were promising sources of high-yielding maize under corn borer infestation and showed relative earliness within their maturity groups.

Sesamia nonagrioides; Ostrinia nubilalis; Corn borer; Zea mays; Core collection; Germplasm

\section{Introduction}

Maintaining genetic resources is a priority in a world concerned with the loss of genetic variability. However, as several authors have pointed out (Goodman, 1990; Shands, 1990; Crossa et al., 1994), ex situ genetic resource management should not just pursue the acquisition and storage of accessions, but go further since the final goal is to use the genetic diversity. Therefore, it would be necessary to characterize, regenerate, and evaluate crop accessions before using them in breeding programs (Crossa et al., 1994). Germplasm from a representative subset could be used to facilitate evaluations while maintaining genetic variation. Frankel and Brown (1984) defined a core collection as the small subset of accessions that represents a large part of the diversity found in the entire collection. Forty-five accessions and three accession composites were selected from a total of 848 Tuxpeño accessions and accession composites to form the final core subset of the maize race Tuxpeño (Taba et al., 1992; Crossa et al., 1994). A core subset of the Caribbean maize collection was made by selecting the upper $20 \%$ of the accessions (100 in total) which represent the phenotypic diversity and have superior selection indexes based on yield, ear rot, plant erectness, and moisture (Taba et al., 1998). Several European maize breeding groups have a commitment with the European Union to develop the European Union Maize Landrace Core Collection (EUMLCC), evaluate it, and make it available. Several maize landraces from Italy, Germany, France, Greece, Portugal, and Spain have been molecularly, biochemically, and morphologically characterized. A core subset of the total accessions was selected based on those data and breeders' knowledge and will constitute the EUMLCC (Rebourg et al., 2003). Information about those accessions is available at www.montpellier.inra.fr/gap/resgen88/results.htm\#database. 
The EUMLCC should be an active collection that might be largely used in the future by maize breeders. Therefore, it would be advisable to know the performance of these materials under the stress conditions that could happen in the proposed area. There are two main maize pests in central and southern Europe: Ostrinia nubilalis and Sesamia nonagrioides. The former pest is present all across Europe, while the presence of $S$. nonagrioides is limited to the Mediterranean area where it is the main biotic constraint for achieving maize yield potential (Anglade, 1972). The two species are corn borers, whose larvae feed on the pith of maize stalks, but they can also be found on the ears (Butrón et al., 1998; Velasco et al., 1999b).

Efforts have been made to look for sources of resistance to corn borer attack among field corn landraces (Anglade, 1961; Anglade and Bertin, 1968; Guthrie and Dicke, 1972; Hudon and Chiang, 1985 and Hudon and Chiang, 1991; Malvar et al., 1993 and Malvar et al., 2004; Cartea et al., 1994; Butrón et al., 1999a) and sweet corn genotypes (Pounders et al., 1975; Andrew and Carlson, 1976; Grier and Davis, 1980; Velasco et al., 1999a and Velasco et al., 1999b). Nevertheless, few studies have been focused on finding sources of tolerance to S. nonagrioides (Butrón et al., 1998) and O. nubilalis (Anglade et al., 1996). Butrón et al. (1998) found out that only a small part of the variation of yield loss could be predicted from the level of antibiosis against $S$. nonagrioides attack. These findings stressed the importance of selecting genotypes by a comprehensive measurement such as yield loss that combines antibiosis and tolerance. Yield losses, however, could be compensated in part by high potential yield (Lynch, 1980; Butrón et al., 1999b). Therefore, evaluating yield under infestation conditions appears to be the best way to estimate the defense level against insect attack. Selecting for yield under infestation would increase resistance and/or tolerance, depending on the mechanism working against corn borer attack. In addition, it would eliminate the risk of loosing yielding ability after selecting for insect resistance as some authors have reported (Russell et al., 1979; Klenke et al., 1986 and Klenke et al., 1988; Butrón et al., 2000 and Butrón et al., 2002).

This paper complements the information published on the differences among the European maize landraces of the EUMLCC for plant resistance measured as tunnel length in the stem and damage on the grain (Malvar et al., 2004) by evaluating the 
EUMLCC for yield under artificial infestation conditions with either O. nubilalis or $S$. nonagrioides.

\section{Materials and methods}

Eighty-five maize landraces (open-pollinated farmer varieties that are no longer cultivated), that represent maize variability in several European countries, were evaluated under artificial infestation with $S$. nonagrioides and O. nubilalis eggs (Table 2). Landraces were classified into four maturity groups, according to the growing degree units in their countries of origin, and each maturity group was evaluated separately, although checks were the same (hybrids Antares, Costanza, and DK-485). A split-plot design was used to evaluate landraces within each germplasm group. Species of insects (S. nonagrioides or O. nubilalis) were assigned to main plots and landraces to subplots. In the $O$. nubilalis and late variety groups, landrace subplots were sorted in three completely random blocks. Early variety subplots were arranged in a triple lattice $6 \times 6$, while midseason variety subplots were arranged in a triple lattice $5 \times 6$. Each experimental subplot consisted of two rows with 25 two-kernel hills. Rows were spaced $0.80 \mathrm{~m}$ apart and hills in the row were spaced $0.21 \mathrm{~m}$ apart. After thinning, the final density was approximately 60,000 plants $\mathrm{ha}^{-1}$.

Evaluations were made at two locations, Pontevedra and Zaragoza, in 2001. The places used for testing are both in Spain but their climatic conditions are totally different. $S$. nonagrioides is the most abundant corn borer in Pontevedra, while European corn borer is predominant in Zaragoza (Malvar et al., 1993; Cordero et al., 1998). However, artificial infestations with both borers were made at both locations. Zaragoza $\left(41^{\circ} 44^{\prime} \mathrm{N}, 0^{\circ} 47^{\prime} \mathrm{W}\right)$ is an inland location characterized by very cold winters and warm summers, while Pontevedra $\left(42^{\circ} 30^{\prime} \mathrm{N}, 8^{\circ} 46^{\prime} \mathrm{W}\right)$ is on the Atlantic coast and temperatures are mild all year around. Therefore, Zaragoza has a continental climate and Pontevedra has a climate with some Mediterranean characteristics. In addition, ecological considerations were taken into account for selecting those locations, because evaluations were made under infestation with two borer species and we did not want to spread any of them in areas where they are not naturally present (in northern and central Europe $S$. nonagrioides is not present). Under infestation conditions, in general, non-significant year×genotype interactions were previously found for any damage trait (Butrón et al., 1999a and Butrón et al., 1998; Velasco et al., 1999b). As significant year×genotype 
interactions were not expected and the insect rearing and infestations consume time, work, and cost, the evaluation was only made in one growing season. Besides, materials evaluated are not breeding populations but landraces and this work constitutes a preliminary study on the abilities of these populations for being used for breeding purposes, and to discard those populations with very poor agronomic characteristics.

O. nubilalis and S. nonagrioides were infested as eggs during silking stage of corn. Ten plants per subplot received a mass of about 40 eggs of either $S$. nonagrioides or $O$. nubilalis, depending on the main plot. The infestation was made according to Butrón et al. (1998): eggs were placed between the shank of main ear and the stem. The rearing method of S. nonagrioides has been described by Eizaguirre (1989). The O. nubilalis eggs were supplied by the Centre de Recherches de Poitou-Charentes (Institute National de la Recherche Agronomique, France).

In each subplot, number of days to silking (from sowing to $50 \%$ plants silking) and percentage of grain moisture at harvest were recorded. Yield of infested plants at $140 \mathrm{~g} \mathrm{~kg}^{-1}$ moisture content was calculated from the ears of infested plants.

Individual and combined analyses of variance over locations were calculated for yield of infested plants, grain moisture, and days to silking. Locations and replications were considered as random factors and landraces and species of insect as fixed factors. Comparisons of means were carried out by the least significant difference (LSD) method. All analyses were made using the SAS software (SAS Institute, 2000).

\section{Results and discussion}

There were not significant differences between species of insect for yield of infested plants, and the insect species x variety interaction was non-significant for any maturity group (Table 1), so performance of maize varieties under infestation by $S$. nonagrioides and O. nubilalis was similar. Velasco et al. (1999b) had already pointed out that resistance to both corn borers is not completely independent.

Table 1.

Mean squares of the analysis of variance of the EUMLCC for three traits evaluated under corn borer infestation with S. nonagrioides and O. nubilalis in 2001 


\begin{tabular}{|c|c|c|c|c|}
\hline Source of variation & df & Yield & Grain moisture & Days to silking \\
\hline \multicolumn{5}{|l|}{ Extra-early } \\
\hline Location (L) & 1 & 46.93 & $2787.60^{*}$ & $414.82^{*}$ \\
\hline Insect (I) & 1 & 5.30 & 43.57 & 63.56 \\
\hline$L \times 1$ & 1 & 7.64 & $22.92^{*}$ & 6.82 \\
\hline Replication (L×I) & 8 & $2.23^{* *}$ & 4.30 & $6.94^{* * *}$ \\
\hline Variety (V) & 12 & $12.06^{* *}$ & $167.79^{* * *}$ & $747.79^{* *}$ \\
\hline$L \times V$ & 10 & 1.32 & $9.50^{* * *}$ & 10.93 \\
\hline $1 \times V$ & 12 & 0.48 & $5.77^{*}$ & 5.89 \\
\hline$L \times \mid \times V$ & 10 & 0.88 & 1.71 & $6.53^{* * *}$ \\
\hline Error & 87 & 0.55 & 2.30 & 2.48 \\
\hline \multicolumn{5}{|l|}{ Early } \\
\hline Location (L) & 1 & 154.62 & $4892.21^{*}$ & 476.10 \\
\hline Insect (I) & 1 & 0.04 & 124.52 & 6.05 \\
\hline$L \times \mid$ & 1 & $15.35^{* * *}$ & 35.78 & 16.04 \\
\hline Replication (L×I) & 8 & 0.46 & $8.15^{* * *}$ & $8.10^{* * *}$ \\
\hline Variety (V) & 32 & $11.94^{* * *}$ & $47.92^{* *}$ & $59.50^{* *}$ \\
\hline$L \times V$ & 29 & $3.46^{* *}$ & $14.90^{*}$ & $5.48^{\text {*** }}$ \\
\hline $\mid \times V$ & 32 & 0.54 & 2.80 & 1.93 \\
\hline$L \times \mid \times V$ & 29 & $1.02^{* *}$ & 3.04 & 2.24 \\
\hline Error & 244 & 0.50 & 3.12 & 1.57 \\
\hline \multicolumn{5}{|l|}{ Midseason } \\
\hline Location (L) & 1 & $202.90^{*}$ & $7914.80^{\text {*交 }}$ & $1465.57^{* \text { * }}$ \\
\hline Insect (I) & 1 & 0.62 & 37.02 & 13.74 \\
\hline$L \times \mid$ & 1 & 4.01 & 5.86 & 5.80 \\
\hline Replication (L×I) & 8 & 1.71 & $3.45^{*}$ & $5.30^{* * *}$ \\
\hline Variety (V) & 26 & $8.64^{* *}$ & $30.35^{* * *}$ & $79.44^{* *}$ \\
\hline$L \times V$ & 22 & 3.17 & $7.42^{* * *}$ & 19.38 \\
\hline $1 \times V$ & 26 & 0.93 & 1.59 & 12.32 \\
\hline
\end{tabular}




\begin{tabular}{|c|c|c|c|c|}
\hline Source of variation & df & Yield & Grain moisture & Days to silking \\
\hline$L \times \mid \times V$ & 22 & 1.65 & 1.53 & $14.63^{* *}$ \\
\hline Error & 192 & 1.18 & 1.62 & 1.52 \\
\hline \multicolumn{5}{|l|}{ Late } \\
\hline Location (L) & 1 & 0.86 & $1563.45^{\text {t* }}$ & $534.02^{* *}$ \\
\hline Insect (I) & 1 & 0.09 & 11.18 & $2.87^{*+1}$ \\
\hline$L \times 1$ & 1 & 0.34 & 6.25 & 0.42 \\
\hline Replication $(L \times \mid)$ & 8 & 0.84 & 5.53 & 6.76 \\
\hline Variety (V) & 11 & $31.47^{* * *}$ & 64.01 & 127.30 \\
\hline$L \times V$ & 4 & 0.91 & 13.24 & $39.60^{\text {* }}$ \\
\hline $\mathrm{I} \times \mathrm{V}$ & 11 & 1.32 & 3.87 & 2.87 \\
\hline$L \times \mid \times V$ & 4 & 0.95 & 5.55 & 3.67 \\
\hline Error & 59 & 1.89 & 3.75 & 3.23 \\
\hline
\end{tabular}

Significant at the $5 \%$ and $1 \%$ level of probability, respectively.

Within the group of early landraces, there were significant interactions between landraces and locations and between landraces, locations, and insects for yield of infested plants (Table 1). Individual analysis of variance of early landraces for yield of infested plants showed that the species of insectxvariety interaction was significant in Zaragoza, while it was not significant in Pontevedra (data not shown). Nevertheless, in Zaragoza, landraces that had a good performance under $O$. nubilalis attack were among the most productive under infestation with $S$. nonagrioides, excepting the variety PRT00100186 (data not shown). Besides, the locationxvariety interaction for yield of infested plants appeared to be mostly due to magnitude changes rather than to changes in rank. For grain moisture, and days to silking, there were significant differences among extra-early, early, and midseason landraces, although some interactions with landraces were significant for those traits.

Among the extra-early landraces, DEU1460239, GRC0010085, ESP0090214 (named 'Viana'), FRA0410010, and ESP0070339 stood out for being significantly more productive than the remaining landraces under artificial infestation (Table 2). However, 
DEU1460239 showed high grain moisture at harvest (25\%) and GRC0010085 flowered even later than the latest check (Costanza). Some mistake classifying landraces into maturity groups, according to the growing degree units in their countries of origin, should be responsible for the inclusion of GRC0010085 in the extra-early group because it flowered as late as landraces included in the late trial. Viana had already performed well under natural infestation in a previous study (Malvar et al., 1993). The Landrace DEU140023 had a very bad performance because it yielded $0.54 \mathrm{mg} \mathrm{ha}^{-1}$. Therefore, removal from the EUMLCC should be considered.

Table 2.

Kernel characteristics, days to silking, grain moisture, and yield under infestation with $S$. nonagrioides and O. nubilalis of the EUMLCC evaluated at two locations in 2001

\begin{tabular}{|l|l|l|l|l|l|}
\hline $\begin{array}{l}\text { Accession } \\
\text { number }\end{array}$ & $\begin{array}{l}\text { Kernel } \\
\text { type }\end{array}$ & $\begin{array}{l}\text { Kernel } \\
\text { color }\end{array}$ & $\begin{array}{l}\text { Days to } \\
\text { silking } \\
\text { (No.) }\end{array}$ & $\begin{array}{l}\text { Grain } \\
\text { moisture (\%) }\end{array}$ & $\begin{array}{l}\text { Yield of infested } \\
\left.\text { plants (Mg ha } \mathbf{~}^{-1}\right)\end{array}$ \\
\hline Extra-early & \multicolumn{5}{|l|}{} \\
\hline DEU1460023 & Flint & Yellow & 51 & 19.5 & 0.54 \\
\hline DEU1460239 & Flint & Yellow & 59 & 25.2 & 5.12 \\
\hline ESP0070339 & Flint & Yellow & 57 & 19.9 & 4.03 \\
\hline ESP0090214 & Flint & Yellow & 54 & 20.8 & 4.27 \\
\hline ESP0090300 & $\begin{array}{l}\text { Semi- } \\
\text { flint }\end{array}$ & Orange & 50 & 21.1 & 1.90 \\
\hline FRA0410010 & Flint & Yellow & 53 & 19.6 & 4.09 \\
\hline FRA0410031 & Flint & Yellow & 58 & 20.2 & 2.92 \\
\hline FRA0410969 & Flint & Orange & 54 & 18.6 & 3.02 \\
\hline GRC0010085 & $\begin{array}{l}\text { Semi- } \\
\text { flint }\end{array}$ & White & 82 & 32.6 & 4.70 \\
\hline PRT00100088 & Flint & Yellow & 53 & 18.3 & 2.78 \\
\hline PRT00100867 & Flint & Yellow & 60 & 21.1 & 3.38 \\
\hline PRT00100813 & Flint & Yellow & 53 & 21.2 & 2.79 \\
\hline PRT00100916 & Flint & Yellow & 56 & 21.5 & 2.94 \\
\hline
\end{tabular}




\begin{tabular}{|c|c|c|c|c|c|}
\hline $\begin{array}{l}\text { Accession } \\
\text { number }^{\mathrm{a}}\end{array}$ & $\begin{array}{l}\text { Kernel } \\
\text { type }\end{array}$ & $\begin{array}{l}\text { Kernel } \\
\text { color }\end{array}$ & $\begin{array}{l}\text { Days to } \\
\text { silking } \\
\text { (No.) }\end{array}$ & $\begin{array}{l}\text { Grain } \\
\text { moisture (\%) }\end{array}$ & $\begin{array}{l}\text { Yield of infested } \\
\text { plants }\left(\mathrm{Mg} \mathrm{ha}^{-1}\right)\end{array}$ \\
\hline LSD (5\%) & & & 3 & 3.1 & 1.14 \\
\hline Antares & & & 59 & 18.9 & 8.00 \\
\hline Costanza & & & 76 & 31.7 & 14.74 \\
\hline DK-485 & & & 72 & 25.1 & 11.38 \\
\hline \multicolumn{6}{|l|}{ Early } \\
\hline DEU1460158 & Flint & Yellow & 59 & 25.0 & 3.31 \\
\hline DEU1460312 & Flint & Yellow & 61 & 20.6 & 2.63 \\
\hline ESP0070127 & Flint & Brown & 60 & 19.0 & 3.19 \\
\hline ESP0070217 & Flint & Orange & 65 & 22.2 & 4.85 \\
\hline ESP0070892 & Flint & Orange & 63 & 21.6 & 4.82 \\
\hline ESP0090205 & Flint & Yellow & 66 & 23.7 & 6.67 \\
\hline ESP11981040 & Flint & White & 63 & 21.9 & 4.88 \\
\hline ESP11982019 & Flint & Yellow & 61 & 19.8 & 4.76 \\
\hline ESP11982031 & Flint & Yellow & 64 & 22.4 & 5.86 \\
\hline ESP11985022 & Flint & Yellow & 60 & 19.7 & 5.72 \\
\hline FRA0410006 & Flint & Yellow & 60 & 18.8 & 4.50 \\
\hline FRA0410015 & Flint & Yellow & 60 & 20.6 & 4.11 \\
\hline FRA0410022 & Flint & Yellow & 59 & 19.9 & 5.24 \\
\hline FRA0410023 & Flint & Yellow & 59 & 20.0 & 4.01 \\
\hline FRA0410090 & Flint & Brown & 65 & 24.1 & 6.12 \\
\hline FRA0410474 & Flint & White & 62 & 21.2 & 4.38 \\
\hline GRC0010016 & $\begin{array}{l}\text { Semi- } \\
\text { flint }\end{array}$ & Yellow & 67 & 25.2 & 4.24 \\
\hline GRC0010017 & Flint & Yellow & 66 & 23.5 & 3.95 \\
\hline GRC0010051 & Flint & Orange & 62 & 22.6 & 4.79 \\
\hline GRC0010084 & Flint & Yellow & 66 & 24.2 & 6.33 \\
\hline GRC0010160 & Flint & Yellow & 61 & 22.0 & 4.47 \\
\hline
\end{tabular}




\begin{tabular}{|c|c|c|c|c|c|}
\hline $\begin{array}{l}\text { Accession } \\
\text { number }^{\mathrm{a}}\end{array}$ & $\begin{array}{l}\text { Kernel } \\
\text { type }\end{array}$ & $\begin{array}{l}\text { Kernel } \\
\text { color }\end{array}$ & $\begin{array}{l}\text { Days to } \\
\text { silking } \\
\text { (No.) }\end{array}$ & $\begin{array}{l}\text { Grain } \\
\text { moisture (\%) }\end{array}$ & $\begin{array}{l}\text { Yield of infested } \\
\text { plants }\left(\mathrm{Mg} \mathrm{ha}^{-1}\right)\end{array}$ \\
\hline GRC0010172 & $\begin{array}{l}\text { Semi- } \\
\text { dent }\end{array}$ & White & 64 & 23.7 & 4.15 \\
\hline GRC0010179 & Flint & Orange & 60 & 21.5 & 2.45 \\
\hline ITA0370071 & $\begin{array}{l}\text { Semi- } \\
\text { flint }\end{array}$ & Orange & 60 & 24.5 & 4.78 \\
\hline ITA0370154 & Flint & Orange & 64 & 23.5 & 4.51 \\
\hline PRT00100019 & Flint & Yellow & 65 & 26.0 & 4.52 \\
\hline PRT00100049 & Flint & Yellow & 66 & 22.9 & 4.68 \\
\hline PRT00100120 & Flint & Yellow & 61 & 20.8 & 4.55 \\
\hline PRT00100186 & Flint & Yellow & 63 & 20.8 & 5.24 \\
\hline PRT00100291 & Flint & White & 63 & 18.3 & 5.05 \\
\hline PRT00100394 & Flint & Yellow & 62 & 21.1 & 5.14 \\
\hline PRT00100815 & Flint & Yellow & 61 & 24.1 & 4.33 \\
\hline PRT00100828 & Flint & Yellow & 63 & 17.6 & 2.68 \\
\hline LSD (5\%) & & & 2 & 3.4 & 1.62 \\
\hline Antares & & & 60 & 18.9 & 7.61 \\
\hline Costanza & & & 77 & 32.3 & 14.46 \\
\hline genbank:DK-465 & & & 71 & 23.6 & 9.38 \\
\hline \multicolumn{6}{|l|}{ Midseason } \\
\hline ESP0070784 & Flint & Yellow & 68 & 23.1 & 5.48 \\
\hline ESP0090025 & Dent & Yellow & 66 & 21.7 & 4.26 \\
\hline ESP0090033 & $\begin{array}{l}\text { Semi- } \\
\text { dent }\end{array}$ & Yellow & 66 & 20.9 & 5.23 \\
\hline ESP0090067 & Flint & Yellow & 71 & 20.3 & 4.64 \\
\hline ESP0090343 & Dent & Yellow & 71 & 25.5 & 4.95 \\
\hline ESP11973C03 & Dent & Yellow & 68 & 20.9 & 6.16 \\
\hline ESP11981047 & $\begin{array}{l}\text { Semi- } \\
\text { Dent }\end{array}$ & Yellow & 64 & 20.7 & 5.51 \\
\hline ESP11982012 & Flint & Yellow & 65 & 22.2 & 5.61 \\
\hline
\end{tabular}




\begin{tabular}{|c|c|c|c|c|c|}
\hline $\begin{array}{l}\text { Accession } \\
\text { number }^{a}\end{array}$ & $\begin{array}{l}\text { Kernel } \\
\text { type }\end{array}$ & $\begin{array}{l}\text { Kernel } \\
\text { color }\end{array}$ & $\begin{array}{l}\text { Days to } \\
\text { silking } \\
\text { (No.) }\end{array}$ & $\begin{array}{l}\text { Grain } \\
\text { moisture (\%) }\end{array}$ & $\begin{array}{l}\text { Yield of infested } \\
\text { plants }\left(\mathrm{Mg} \mathrm{ha}^{-1}\right)\end{array}$ \\
\hline ESP11985020 & Flint & White & 65 & 22.2 & 6.66 \\
\hline FRA0410194 & $\begin{array}{l}\text { Semi- } \\
\text { flint }\end{array}$ & Yellow & 66 & 22.6 & 6.68 \\
\hline FRA0410496 & Flint & White & 71 & 20.1 & 4.74 \\
\hline FRA0410619 & Flint & Yellow & 66 & 20.4 & 4.96 \\
\hline FRA0410625 & Flint & Yellow & 73 & 21.1 & 4.77 \\
\hline FRA0410636 & Flint & White & 73 & 20.1 & 3.37 \\
\hline FRA0410639 & Flint & White & 69 & 21.9 & 5.96 \\
\hline FRA0410668 & Flint & White & 69 & 22.1 & 5.39 \\
\hline GRC0010012 & Flint & Yellow & 70 & 21.9 & 5.33 \\
\hline GRC0010165 & $\begin{array}{l}\text { Semi- } \\
\text { dent }\end{array}$ & Yellow & 68 & 24.9 & 4.96 \\
\hline GRC0010174 & $\begin{array}{l}\text { Semi- } \\
\text { dent }\end{array}$ & White & 65 & 22.7 & 5.67 \\
\hline GRC0010183 & Flint & White & 67 & 21.5 & 4.36 \\
\hline ITA0370058 & $\begin{array}{l}\text { Semi- } \\
\text { flint }\end{array}$ & Yellow & 65 & 23.1 & 6.41 \\
\hline ITA0370143 & $\begin{array}{l}\text { Semi- } \\
\text { flint }\end{array}$ & Red & 63 & 24.2 & 5.98 \\
\hline ITA0370185 & Dent & White & 67 & 29.1 & 9.05 \\
\hline ITA0370488 & $\begin{array}{l}\text { Semi- } \\
\text { flint }\end{array}$ & Orange & 65 & 24.6 & 5.77 \\
\hline PRT00100392 & Flint & White & 63 & 20.3 & 6.17 \\
\hline PRT00101526 & Flint & White & 65 & 18.8 & 4.18 \\
\hline PRT00102047 & Flint & White & 70 & 21.5 & 5.67 \\
\hline LSD (5\%) & & & 4 & 2.5 & 1.61 \\
\hline Antares & & & 60 & 18.0 & 7.82 \\
\hline Costanza & & & 77 & 26.7 & 13.52 \\
\hline DK-485 & & & 71 & 20.8 & 10.29 \\
\hline
\end{tabular}




\begin{tabular}{|l|l|l|l|l|l|}
\hline $\begin{array}{l}\text { Accession } \\
\text { number }\end{array}$ & $\begin{array}{l}\text { Kernel } \\
\text { type }\end{array}$ & $\begin{array}{l}\text { Kernel } \\
\text { color }\end{array}$ & $\begin{array}{l}\text { Days to } \\
\text { silking } \\
\text { (No.) }\end{array}$ & $\begin{array}{l}\text { Grain } \\
\text { moisture (\%) }\end{array}$ & $\begin{array}{l}\text { Yield of infested } \\
\left.\text { plants } \mathbf{M g ~ h a ~}^{-1}\right)\end{array}$ \\
\hline Late & \multicolumn{5}{|l|}{} \\
\hline DEU1460013 & Dent & Yellow & 68 & 29.1 & 2.64 \\
\hline ESP0070441 & Pop & Purple & 82 & 24.3 & 3.27 \\
\hline ESP0090032 & Dent & Yellow & 71 & 23.6 & 6.30 \\
\hline ESP0090315 & $\begin{array}{l}\text { Semi- } \\
\text { dent }\end{array}$ & White & 78 & 24.6 & 4.48 \\
\hline ITA0370005 & Flint & Orange & 70 & 27.8 & 6.68 \\
\hline ITA0370026 & Dent & White & 69 & 29.7 & 6.51 \\
\hline ITA0370088 & Dent & Yellow & 69 & 31.9 & 4.53 \\
\hline ITA0370100 & Flint & White & 72 & 33.2 & 7.34 \\
\hline ITA0370171 & Dent & Yellow & 72 & 32.5 & 8.67 \\
\hline ITA0370195 & $\begin{array}{l}\text { Semi- } \\
\text { floury }\end{array}$ & Yellow & 70 & 30.3 & 5.34 \\
\hline PRT00100530 & Flint & Orange & 78 & 29.5 & 7.58 \\
\hline PRT00100569 & Flint & Yellow & 80 & 31.3 & 8.17 \\
\hline LSD (5\%) & & & & & 1.36 \\
\hline Antares & & & 60 & 18.2 & 7.32 \\
\hline Costanza & & & 78 & 26.5 & 12.26 \\
\hline DK-485 & & & 71 & 20.9 & 9.53 \\
\hline The first & & & & & \\
\hline
\end{tabular}

${ }^{a}$ The first three letters stand for the country of origin (DEU for Germany, ESP for Spain, FRA for France, GRC for Greece, ITA for Italy, and PRT for Portugal).

Among the early landraces, ESP0090205, GRC00110084, FRA0410090, ESP11982031, ESP11985022, FRA0410022, FRA0410022, PRT00100186, and PRT00100394 had the greatest yields under infestation conditions. However, as we previously mentioned, PRT00100186 had poor yielding ability under artificial infestation with S. nonagrioides eggs in Zaragoza. ESP0090205 (named 'Tuy') was previously tested under natural infestation and was among the most productive landraces (Ordás et al., 1988; Malvar et al., 1993). FRA0410022 and ESP11985022, besides being productive under infestation 
conditions, were very early. Landraces GRC0010084, FRA0410090, and ESP0090205, however, presented days to flowering and grain moisture at harvest significantly higher than FRA0410022 and ESP11985022. Therefore, landraces as FRA0410022 and ESP11985022 could be used for developing early materials, while GRC0010084, FRA0410090, and ESP0090205 could be the base materials for growing areas with longer growing season.

The variety ITA0370185 was the highest yielding among the midseason landraces. However, Italian landraces were only evaluated in one environment (Pontevedra) because not enough seed was available to perform two trials. Therefore, it is necessary to evaluate those landraces in at least on other environment to have more reliable data about their performance under corn borer infestation. Besides, in Pontevedra, other midseason varieties such as ESP11985020, PRT00100392, FRA0410194, ESP11973C03, and PRT00102047 did not significantly differ from the Italian landrace ITA03700185 (data not shown). In the midseason group, among landraces evaluated in two environments, FRA0410194, ESP11985020, PRT00100392, ESP11973C03, FRA0410639, GRC0010174, PRT00102047, ESP11982012, ESP11981047, ESP0070784, FRA0410668, GRC0010012, and ESP0090033 were significantly more productive under corn borer infestations. Landraces PRT00100392 and ESP11981047 showed less grain moisture and days to silking than others. The population ESP0090033 (named 'Tremesino') had been already cited as a promising source of yielding ability under corn borer infestation because it had favorable variety effects for yield under infestation with S. nonagrioides in a previous study (Soengas et al., 2004).

In the late group, landraces ITA0370171, PRT00100569, PRT00100530, and ITA0370100 performed better than others under corn borer infestation conditions. However, Italian landraces, as we have already said, were tested in one environment only. The variety ESP0090032 (named 'Rastrojero'), that in previous studies was among the most resistant and productive populations under corn borer natural infestation conditions (Ordás et al., 1988; Malvar et al., 1993), performed significantly worse than PRT00100569 under artificial infestation conditions. Therefore, this work was able to find materials even more suitable to use in breeding programs to decrease corn borer attack impact than the ones that we already had. It would be necessary to study the heterosis among these promising populations, to know the real potential of these populations for 
generating inbred lines that in hybrid combination will render productive hybrids under corn borer infestations. A study on heterosis patterns among French and Spanish populations reported that populations Lazcano, from northern Spain, and Millete de Lauragais, from southern France, would be appropriate for developing inbred lines with high heterosis effects when crossed (Malvar et al., 2004). Millete de Lauragais is a midseason population (FRA0410639) that had good performance under corn borer infestation.

In general, the most productive landraces under artificial infestation with corn borers came from southern Europe, as expected, because they should be adapted to the high natural infestation, mainly by $S$. nonagrioides, that is a common pest of maize in that region. In general, the most productive landraces under corn borer infestation had their origin in areas with mild temperatures all throughout the year. In contrast, in Germany, northern France, or Central Spain where cold winter temperatures limit corn borer development, few populations were reported with high yield under infestation conditions. Ordás et al. (1988) found out that US Corn Belt populations were less resistant than Spanish populations since $O$. nubilalis is a recent pest in USA and $S$. nonagrioides is not present in that country.

In conclusion, yield under infestation conditions, as an indicator of the level of defense against corn borer attack, of 85 maize landraces was evaluated and the most promising sources could be used in breeding programs. Extra-early landraces, ESP0090214, FRA0410010, and ESP0070339; early landraces, FRA0410022 and ESP11985022; midseason landraces, PRT00100392 and ESP11981047; and late landraces, PRT00100569 and PRT00100530, were the most promising sources of high yields under artificial infestation with corn borer eggs and showed relative earliness. Crosses among the inbred lines developed from these populations will follow the heterotic pattern 'flint $\times$ flint' that could be a good alternative to the most common hybrids used in Europe, 'European flint×American dent', mostly in regions where earliness is required.

\section{Acknowledgments}

Research supported by the European Union (RESGEN-CT96-088) and the Excma. Diputación Provincial de Pontevedra, Spain. A. Butrón and G. Padilla acknowledge the 
support that they got from the Spanish Ministry for Science and Technology and the 'Cabildo de la Isla de La Palma', respectively.

\section{References}

Andrew and Carlson, 1976 R.H. Andrew and P.R. Carlson, Evaluation of sweet corn inbreds for resistance for European corn borer, J. Am. Soc. Hort. Sci. 101 (1976), pp. 97-99.

Anglade, 1961 P. Anglade, Essai de mise au point d'une méthode de mesure de la sensibilité des lignées de maïs aux chenilles de la génération estivale de la Sésamie (Sesamia nonagrioides Lef.), Ann. Epiph. 12 (1961), pp. 413-422.

Anglade, 1972 P. Anglade, Les Sesamia. In: A.S. Balachowsky, Editor, Entomologie appliquée à l'agriculture. II, Lépidoptères, II, Masson et Cie, Paris, France (1972), pp. 1389-1400.

Anglade and Bertin, 1968 P. Anglade and C. Bertin, Mise en évidence d'une résistance à la Sésamie des lignées de maïs et de sa transmission aux hybrides, Ann. Epiph. 19 (1968), pp. 579-587.

Anglade et al., 1996 P. Anglade, B. Gouesnard, A. Boyat and A. Panouillé, Effects of multitrait recurrent selection for ECB tolerance and for agronomic traits in FS12 maize synthetic, Maydica 41 (1996), pp. 97-104.

Butrón et al., 1998 A. Butrón, R.A. Malvar, P. Velasco, P. Revilla and A. Ordás, Defense mechanisms of maize against pink stem borer, Crop Sci. 38 (1998), pp. 1159-1163.

Butrón et al., 1999a A. Butrón, R.A. Malvar, M.E. Cartea, A. Ordás and P. Velasco, Resistance of maize inbreds to pink stem borer, Crop Sci. 39 (1999), pp. 102-107.

Butrón et al., 1999b A. Butrón, R.A. Malvar, P. Velasco, M.I. Vales and A. Ordás, Combining abilities for maize stem antibiosis, yield loss and yield under infestation and non infestation with pink stem borer, Crop Sci. 39 (1999), pp. 691-696

Butrón et al., 2000 A. Butrón, N.W. Widstrom, M.E. Snook and B.R. Wiseman, Recurrent selection for corn earworm resistance in three corn synthetics, Maydica 45 (2000), pp. 295-300. 
Butrón et al., 2002 A. Butrón, N.W. Widstrom, M.E. Snook and B.R. Wiseman, Recurrent selection for corn earworm (Lepidoptera: Noctuidae) resistance in three closely related corn southern synthetics, J. Econ. Entomol. 95 (2002), pp. 458-462.

Cartea et al., 1994 M.E. Cartea, R.A. Malvar, P. Revilla, A. Ordás and A. Álvarez, Seasonal occurrence and response of maize inbred lines to pink stem borer in the northwest of Spain, Maydica 39 (1994), pp. 191-196.

A. Cordero, R.A. Malvar, A. Butrón, P. Revilla, P. Velasco and A. Ordás, Population dynamics and life-cycle of corn borers in south Atlantic European coast, Maydica 43 (1998), pp. 5-12.

Crossa et al., 1994 J. Crossa, S. Taba, S.A. Eberhart, P. Bretting and R. Vencovsky, Practical considerations for maintaining germplasm in maize, Theor. Appl. Genet. 89 (1994), pp. 89-95.

Eizaguirre, 1989 Eizaguirre, M., 1989. Inducción de la diapausa en Sesamia nonagrioides Lef. (Lepidoptera: Noctuidae) y su papel en el ciclo biológico de las comarcas de Lérida. Ph.D. Thesis. Universidad Politécnica de Catalunya, Spain.

Frankel and Brown, 1984 O.H. Frankel and A.H.D. Brown, Current plant genetic resources: a critical appraisal, Genetics: New Frontiers vol. IV, Oxford and IBH Publ. Co., New Delhi, India (1984), pp. 1-11.

Goodman, 1990 M.M. Goodman, Genetic and germplasm stocks worth conserving, J. Heredity 81 (1990), pp. 11-16.

Grier and Davis, 1980 S.L. Grier and D.W. Davis, Infestation procedures and heritability of characters used to estimate ear damage caused by second-brood European corn borer (O. nubilalis, Hübner) on corn, J. Am. Soc. Hort. Sci. 105 (1980), pp. 3-8.

Guthrie and Dicke, 1972 W.F. Guthrie and F.F. Dicke, Resistance of inbreds lines of dent corn to leaf feeding by first-brood European corn borer, lowa State J. Sci. 46 (1972), pp. 339-357.

Hudon and Chiang, 1985 M. Hudon and M.S. Chiang, Resistance and tolerance of maize germplasm to the European corn borer $O$. nubilalis (Hübner) and its maturity in Quebec, Maydica 30 (1985), pp. 329-337. 
Hudon and Chiang, 1991 M. Hudon and M.S. Chiang, Evaluation of resistance of maize germplasm to the univoltine European corn borer $O$. nubilalis (Hübner) and relationship with maize maturity in Quebec, Maydica 36 (1991), pp. 69-74.

Klenke et al., 1986 J.R. Klenke, W.A. Russell and W.D. Guthrie, Recurrent selection for resistance to European corn borer in a corn synthetic and correlated effects on agronomic traits, Crop Sci. 26 (1986), pp. 864-868.

Klenke et al., 1988 J.R. Klenke, W.A. Russell, W.D. Guthrie and O.S. Smith, Inbreeding depression and gene frequency changes for agronomic traits in corn synthetic selected for resistance to European corn borer, J. Agric. Entomol. 5 (1988), pp. 225-233.

Lynch, 1980 R.E. Lynch, European corn borer: yield loss in relation to hybrid and stage of corn development, J. Econ. Entomol. 73 (1980), pp. 159-164.

Malvar et al., 1993 R.A. Malvar, M.E. Cartea, P. Revilla, A. Ordás, A. Álvarez and J.P. Mansilla, Sources of resistance to pink stem borer and European corn borer in maize, Maydica 38 (1993), pp. 313-319.

Malvar et al., 2004 R.A. Malvar, A. Butrón, A. Álvarez, B. Ordás, P. Soengas, P. Revilla and A. Ordás, Evaluation of the European Union maize landrace core collection for resistance to Sesamia nonagrioides (Lepidoptera: Noctuidae) and O. nubilalis (Lepidoptera: Crambidae), J. Econ. Entomol. 97 (2004), pp. 628-634.

Ordás et al., 1988 A. Ordás, A. Álvarez and R.A. Malvar, Variability of resistance to the second generation of European corn borer in maize, Maydica 33 (1988), pp. 27-36.

Pounders et al., 1975 C.T. Pounders Jr., D.W. Davis, M.B. Windels and H.C. Chiang, Resistance to second-brood European corn borer attack in dent inbred 'B52' and in progeny from crosses with four sweet corn inbreds, J. Am. Soc. Hort. Sci. 100 (1975), pp. 101-103.

Rebourg et al., 2003 C. Rebourg, M. Chastanet, B. Gouesnard, C. Welcker, P. Dubreuil and $A$. Charcosset, Maize introduction into Europe: the history reviewed in the light of molecular data, Theor. Appl. Genet. 106 (2003), pp. 895-903

Russell et al., 1979 W.A. Russell, G.D. Lawrance and W.D. Guthrie, Effects of recurrent selection for European corn borer resistance on other agronomic characters in synthetic cultivars of corn, Maydica 24 (1979), pp. 33-47. 
SAS Institute and Inc., 2000 SAS Institute, Inc., SAS Online Doc, Version 8, SAS Institute, Inc., Cary, NC (2000).

Shands, 1990 H.L. Shands, Plant genetic resources conservation: the role of the gene bank in delivering useful genetic materials to the research scientist, J. Hered. 81 (1990), pp. 7-10. Soengas et al., 2004 P. Soengas, A. Butrón, P. Revilla, A. Ordás and R.A. Malvar, Performance of crosses among flint maize populations under infestation by Sesamia nonagrioides (Lepidoptera: Noctuidae), J. Econ. Entomol. 97 (2004), pp. 14381443.

Taba et al., 1992 Taba, S., Pineda, F., Crossa, J., 1992. Forming core subsets from the Tuxpeño race complex. Abstracts of the First International Crop Science Congress, Ames, IA, p. 84.

Taba et al., 1998 S. Taba, J. Díaz, J. Franco and J. Crossa, Evaluation of Caribbean maize accessions to develop a core subset, Crop Sci. 38 (1998), pp. 1378-1386

P. Velasco, R.A. Malvar, A. Butrón, P. Revilla and A. Ordás, Ear feeding resistance of sweet corn inbreds to pink stem borer, J. Am. Soc. Hort. Sci. 124 (1999), pp. 268-272

P. Velasco, R.A. Malvar, P. Revilla, A. Butrón and A. Ordás, Ear resistance of sweet corn populations to Sesamia nonagrioides (Lepidoptera:Noctuidae) and O. nubilalis (Lepidoptera: Pyralidae), J. Econ. Entomol. 92 (1999), pp. 732-739. 